\title{
Particle Swarm Optimisation Aided Minimum Bit Error Rate Multiuser Transmission
}

\author{
W. Yao, S. Chen, S. Tan and L. Hanzo \\ School of ECS, University of Southampton, SO17 1BJ, United Kingdom \\ E-mails: \{wy07r, sqc, st104r, lh\}@ecs.soton.ac.uk, http://www-mobile.ecs.soton.ac.uk
}

\begin{abstract}
We consider the downlink of multiuser system from a transmitter equipped with multiple antennas to multiple non-cooperative single-antenna mobile receivers. Particle swarm optimisation (PSO) algorithm is invoked to solve the constrained nonlinear optimisation problem for the minimum bit error rate (MBER) multiuser transmission (MUT). The proposed PSO aided MBER-MUT scheme provides much better performance over the conventional minimum mean-square-error MUT scheme, and it achieves a much lower complexity compared to the state-of-theart sequential quadratic programming based MBER MUT.
\end{abstract}

\section{INTRODUCTION}

For the downlink of a space-division multiple-access (SDMA) system with decentralised non-cooperative mobile devices at the receive end, the mobile users are unable to do cooperative multiuser detection (MUD). In order to achieve better performance, the signals should be pre-processed at the base station (BS), leading to multiuser transmission (MUT). MUT techniques enable the implementation of low-complexity and high power-efficiency mobile terminals (MTs) for mobile broadcast channels when channel state information (CSI) is available at the transmitter [1]. The assumption that the downlink channel impulse response is known at the BS is valid in time division duplex (TDD) systems due to the channel reciprocity. Transmit preprocessing is then possible when the channel coherence time is large compared to one transmission interval. However, for frequency division duplex (FDD) systems, where the uplink and downlink channels are not reciprocal, feedback from the MT receivers to the BS transmitter is necessary [2].

Most of the MUT techniques are based on the minimum mean-square-error (MMSE) criterion [2], [3]. Since the bit error rate (BER) is the ultimate system performance indicator, interests on minimum BER (MBER) based MUT techniques have increased recently. A MBER-MUT scheme was proposed in [4] for the TDD code-division multiple-access (CDMA) downlink over frequency-selective channels, and this work was extended to multiple transmit and receive antennas in [5]. A chip-level MBER-MUT scheme was proposed in [6]. These MBER-MUT techniques [4]-[7] were developed based on the fact that the information symbols to be transmitted are known exactly at the transmitter and the precoding matrix can be chosen specifically for the given symbol vector so that the BER is minimised. For this reason, we refer to this design as symbol-specific MBER MUT. However, the complexity of this symbol-specific MBER MUT design may become prohibitive because the coefficient matrix of the precoder has to be optimised for every transmit symbol vector. An average MBER-MUT design was proposed and investigated for binary phase shift keying (BPSK) modulation [8] and quadrature phase shift keying (QPSK) modulation [9], where the precoder's coefficient matrix only needs to be re-calculated when the channel has changed. Both the symbol-specific and average MBER-MUT designs lead to a constrained nonlinear optimisation [7]-[9], and the sequential quadratic programming (SQP) algorithm [10] is typically used to obtain the precoder's coefficients for the MBER-MUT [7]-[9]. However, the computational complexity of SQP based optimisation may be too high for real-time implementation in high-rate systems, even for the average MBER-MUT design.

In this contribution, we invoke the particle swarm optimisation (PSO) algorithm [11] to find the precoder's coefficients in order to reduce the computational complexity of the MBERMUT. PSO is a population based stochastic optimisation technique [11], [12] inspired by social behaviour of bird flocking or fish schooling. The algorithm starts with random initialisation of a swarm of individuals, called particles, within the problem search space. It then endeavours to find a global optimal solution by adjusting the trajectory of each individual toward its own best location and toward the best position of the entire swarm at each evolution step. The PSO method is becoming very popular due to its simplicity in implementation, ability to quickly converge to a reasonably good solution and its robustness against local minima. It has been applied to wide-ranging optimisation problems successfully [12]-[17]. In particular, many researchers have applied PSO techniques to MUD [18]-[23]. However, no work to date has applied the PSO method to MUT design. We will show that the proposed PSO approach is capable of finding the optimal MBER MUT solution at a lower complexity than the benchmark state-ofthe-art SQP based MBER MUT design.

The rest of this contribution is structured as follows. In Section II, the signal model of the downlink SDMA system is introduced. The MBER-MUT strategy is summarised in Section III, while Section IV outlines our proposed PSO assisted MBER-MUT algorithm. Our simulation study is given in Section V, and we conclude the paper in Section VI.

\section{System MOdEL}

The downlink of a SDMA system with decentralised noncooperative mobile devices at the receive end is considered 


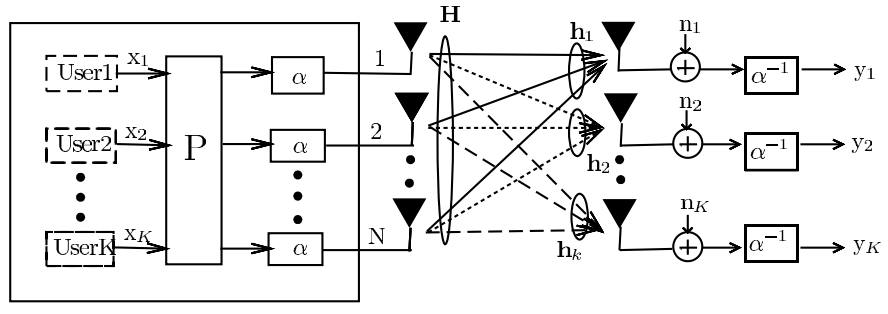

Fig. 1. Schematic diagram of the downlink of a SDMA system using preprocessing at the BS. The system employs $N$ transmit antennas to communicate with $K$ decentralised non-cooperative mobile devices.

here. The BS is equipped with $N$ transmit antennas and communicates over flat fading channels with $K$ MTs, each employing only one receive antenna. Frequency selective channels can be made narrowband using for example the orthogonal frequency division multiplexing (OFDM) technique [24]. This system model is illustrated in Fig. 1. The vector of information symbols for transmission is given by $\mathbf{x}=\left[\begin{array}{ll}x_{1} & x_{2} \cdots x_{K}\end{array}\right]^{T}$, where $x_{k}$ denotes the transmitted symbol for the $k$ th MT and the symbol energy is given by $E\left[\left|x_{k}\right|^{2}\right]=\sigma_{x}^{2}$, for $1 \leq k \leq K$, with $E[\bullet]$ denoting the expectation operator. The $N \times K$ dimensional precoder matrix $\mathbf{P}$ is defined by

$$
\mathbf{P}=\left[\begin{array}{lll}
\mathbf{p}_{1} & \mathbf{p}_{2} \cdots \mathbf{p}_{K}
\end{array}\right]
$$

where $\mathbf{p}_{k}, 1 \leq k \leq K$, is the precoder's coefficient vector for the $k$ th user's data stream. Given a fixed total transmit power $\mathrm{E}_{T}$ at the BS, a scaling factor should be used to fullfill this transmit power constraint, which is defined as

$$
\alpha=\sqrt{\mathrm{E}_{T} / E\left[\|\mathbf{P x}\|^{2}\right]} .
$$

At the receive end, the inverse of the scaling factor, $\alpha^{-1}$, is multiplied with the received signal to ensure unity-gain transmission. The channel matrix $\mathbf{H}$ is given by

$$
\mathbf{H}=\left[\mathbf{h}_{1} \mathbf{h}_{2} \cdots \mathbf{h}_{K}\right]
$$

where $\mathbf{h}_{k}=\left[\begin{array}{ll}h_{1, k} & h_{2, k} \cdots h_{N, k}\end{array}\right]^{T}, 1 \leq k \leq K$, is the $k$ th user's spatial signature. The channel taps $h_{i, k}$ for $1 \leq k \leq K$ and $1 \leq i \leq N$ are independent with each other and obey the complex-valued Gaussian distributions associated with $E\left[\left|h_{i, k}\right|^{2}\right]=1$. The additive Gaussian white noise vector $\mathbf{n}$ is defined by $\mathbf{n}=\left[\begin{array}{ll}n_{1} & n_{2} \cdots n_{K}\end{array}\right]^{T}$, where $n_{k}, 1 \leq k \leq K$, is a complex-valued Gaussian random process with zero mean and $E\left[\left|n_{k}\right|^{2}\right]=2 \sigma_{n}^{2}=\mathrm{N}_{\mathrm{o}}$. The signal-to-noise ratio (SNR) of the downlink is defined as $\mathrm{SNR}=\mathrm{E}_{\mathrm{b}} / \mathrm{N}_{\mathrm{o}}$, where $\mathrm{E}_{\mathrm{b}}=$ $\mathrm{E}_{T} /\left(N \log _{2} M\right)$ is the energy per bit per antenna for $M$-ary modulation. Thus, the baseband model of the system can be described as

$$
\mathbf{y}=\mathbf{H}^{T} \mathbf{P x}+\alpha^{-1} \mathbf{n}
$$

where $\mathbf{y}=\left[\begin{array}{llll}y_{1} & y_{2} & \cdots & y_{K}\end{array}\right]^{T}$ denotes the received signal vector, and $y_{k}, 1 \leq k \leq K$, is a sufficient statistics for the $k$ th MT to detect the transmitted data symbol $x_{k}$.

\section{MBER MULTIUSER TRANSMISSION}

For notational simplicity, we consider the QPSK modulation with $M=4$. Extension to the generic quadrature amplitude modulation can be achieved by considering the minimum symbol error rate criterion, as in the MUD case [25]. To alleviate the computational complexity associated with the symbol-specific MBER MUT scheme, we consider the average MBER MUT design [8], [9], which determines the precoder's matrix that remains optimal for all the legitimate transmission symbol vectors. Without any confusion, it is simply referred to as the MBER MUT design.

The average BER of the in-phase component of $\mathbf{y}$ at the receiver can be shown to be [26]

$$
P_{e_{I}}=\frac{1}{K M^{K}} \sum_{q=1}^{M^{K}} \sum_{k=1}^{K} Q\left(\frac{\operatorname{sgn}\left(\Re\left[x_{k}^{(q)}\right]\right) \Re\left[\mathbf{h}_{k}^{T} \mathbf{P} \mathbf{x}^{(q)}\right]}{\sigma_{n}}\right),
$$

where $M^{K}=4^{K}$ is the number of equiprobable legitimate transmit symbol vectors $\mathbf{x}^{(q)}$ for QPSK signalling, $x_{k}^{(q)}$ the $k$ th element of $\mathbf{x}^{(q)}$, with $1 \leq q \leq M^{K}, Q(\bullet)$ denotes the standard Gaussian error function, and $\Re[\bullet]$ the real part. Similarly, the average BER of the quadrature-phase component of $\mathbf{y}$ is given by [26]

$$
P_{e_{Q}}=\frac{1}{K M^{K}} \sum_{q=1}^{M^{K}} \sum_{k=1}^{K} Q\left(\frac{\operatorname{sgn}\left(\Im\left[x_{k}^{(q)}\right]\right) \Im\left[\mathbf{h}_{k}^{T} \mathbf{P} \mathbf{x}^{(q)}\right]}{\sigma_{n}}\right),
$$

where $\Im[\bullet]$ denotes the imaginary part. Thus the average BER for QPSK signalling is given by

$$
P_{e}(\mathbf{P})=\left(P_{e_{I}}(\mathbf{P})+P_{e_{Q}}(\mathbf{P})\right) / 2
$$

and the solution of the MBER MUT is defined as

$$
\begin{aligned}
& \mathbf{P}_{\mathrm{TxMBER}}=\arg \min _{\mathbf{P}} P_{e}(\mathbf{P}) \\
& \text { s.t. } E\left[\|\mathbf{P} \mathbf{x}\|^{2}\right]=\mathrm{E}_{T} .
\end{aligned}
$$

The optimisation problem (8) is a constrained nonlinear optimisation one, and is typically solved by an iterative gradient based optimisation algorithm known as the SQP [8], [9]. The computational complexity per iteration of the SQP based MBER MUT scheme, taken from [9], is listed in Table I for QPSK modulation, where $\mathcal{O}(\bullet)$ stands for order of • complexity and we assume that the complexity of a real-valued multiplication is equal to a real-valued addition. The total computational complexity equals the number of iterations that the algorithm required to arrive at a global optimal solution multiplied by this complexity per iteration.

\section{PSO ASSISTED MBER MUT}

A penalty function approach is adopted to convert the constrained optimisation (8) into the unconstrained one and to automatically perform power allocation in order to meet the transmit power constraint. Define the cost function

$$
F(\mathbf{P})=P_{e}(\mathbf{P})+G(\mathbf{P})
$$


TABLE I

COMPUTATIONAL COMPLEXITY PER ITERATION OF TWO MBER MUT DESIGN ALGORITHMS FOR QPSK SIGNALLING, WHERE $N$ IS THE NUMBER OF TRANSMIT ANTENNAS, $K$ THE NUMBER OF MOBILE USERS, $M=4$ IS THE SIZE OF SYMBOL CONSTELLATION AND $S$ IS THE SWARM SIZE.

\begin{tabular}{|c|l|}
\hline Algorithm & Flops \\
\hline SQP & $K \times\left(8 \times N^{2} \times K^{2}+6 \times N \times K+6 \times N+8 \times K+4\right) \times M^{K}+\mathcal{O}\left(8 \times N^{3} \times K^{3}\right)+8 \times N^{2} \times K^{2}$ \\
& $+16 \times N \times K^{2}+8 \times N^{2} \times K+12 \times N \times K+6 \times K^{2}-2 \times N^{2}+N-2 \times K+11$ \\
\hline PSO & $\left((16 \times N \times K+7 \times K+6 \times N+1) \times M^{K}+20 \times N \times K+2\right) \times S+8$ \\
\hline
\end{tabular}

with the penalty function given by

$G(\mathbf{P})=\left\{\begin{array}{cc}0, & E\left[\|\mathbf{P x}\|^{2}\right]-\mathrm{E}_{T} \leq 0 \\ \lambda\left(E\left[\|\mathbf{P} \mathbf{x}\|^{2}\right]-\mathrm{E}_{T}\right), & E\left[\|\mathbf{P x}\|^{2}\right]-\mathrm{E}_{T}>0\end{array}\right.$

where the penalty factor $\lambda>0$ should be chosen appropriately so that the MBER-MUT design (8) can be obtained as the solution of the following unconstrained optimisation

$$
\mathbf{P}_{\text {TxMBER }}=\arg \min _{\mathbf{P}}\left\{P_{e}(\mathbf{P})+G(\mathbf{P})\right\} .
$$

We invoke the PSO algorithm to solve this design problem.

The flowchart of the proposed PSO aided MBER-MUT design is given in Fig. 2. A swarm of particles, $\left\{\check{\mathbf{P}}_{i}^{(l)}\right\}_{i=1}^{S}$, that represent potential solutions are evolved in the search space $\mathrm{S}^{N \times K}$, where

$$
\mathrm{S}=\left[-P_{\max }, P_{\max }\right]+j\left[-P_{\max }, P_{\max }\right]
$$

is the square area in the complex plan that defines the search range for each element of the precoder coefficient matrix, $S$ is the swarm size and index $l$ denotes the iteration step.

a) The swarm initialisation. With $l=0$, set $\check{\mathbf{P}}_{1}^{(l)}$ to the MMSE solution, while the rest of the initial particles, $\left\{\check{\mathbf{P}}_{i}^{(l)}\right\}_{i=2}^{S}$, are randomly generated in the search space $\mathrm{S}^{N \times K}$.

b) The swarm evaluation. Each particle $\check{\mathbf{P}}_{i}^{(l)}$ has a cost $F\left(\check{\mathbf{P}}_{i}^{(l)}\right)$ associated with it, which is evaluated according to (9). Each particle $\check{\mathbf{P}}_{i}^{(l)}$ remembers its best position visited so far, denoted as $\mathbf{P} \mathbf{b}_{i}^{(l)}$, which provides the cognitive information. Every particle also knows the best position visited so far among the entire swarm, denoted as $\mathbf{G} \mathbf{b}^{(l)}$, which provides the social

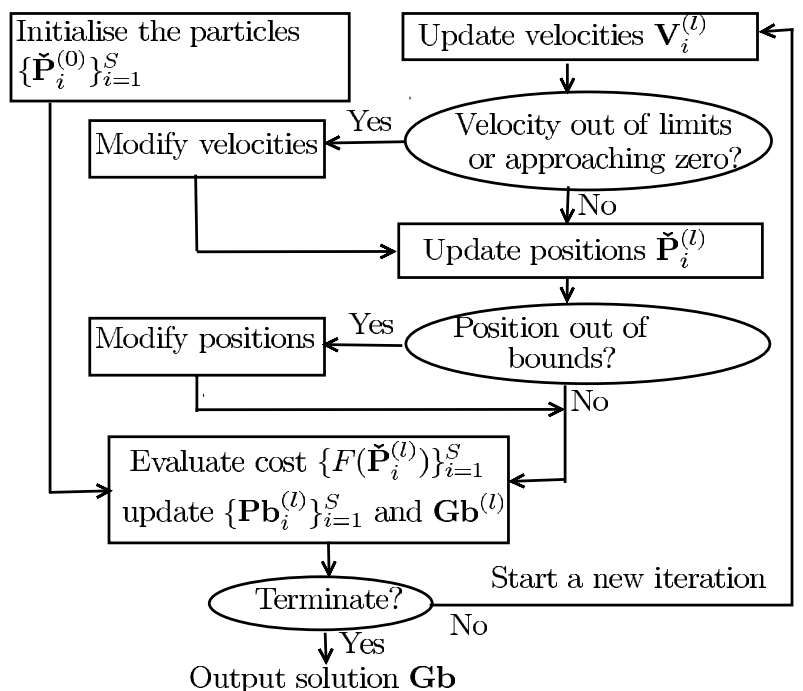

Fig. 2. Flowchart of the particle swarm optimisation algorithm. information. The cognitive information $\left\{\mathbf{P b}_{i}^{(l)}\right\}_{i=1}^{S}$ and the social information $\mathbf{G b}^{(l)}$ are updated at each iteration:

$$
\begin{aligned}
& \text { For }(i=1 ; i<S ; i++) \\
& \quad \text { If }\left(F\left(\check{\mathbf{P}}_{i}^{(l)}\right)<F\left(\mathbf{P b}_{i}^{(l)}\right)\right) \quad \mathbf{P b}_{i}^{(l)}=\check{\mathbf{P}}_{i}^{(l)}
\end{aligned}
$$

End for;

$i^{*}=\arg \min _{1 \leq i \leq S} F\left(\mathbf{P b}_{i}^{(l)}\right)$;

If $\left(F\left(\mathbf{P b}_{i^{*}}^{(l)}\right)<F\left(\mathbf{G b}^{(l)}\right)\right) \quad \mathbf{G b}^{(l)}=\mathbf{P b}_{i^{*}}^{(l)} ;$

c) The swarm update. Each particle $\check{\mathbf{P}}_{i}^{(l)}$ has a velocity, denoted as $\mathbf{V}_{i}^{(l)}$, to direct its "flying". The velocity and position of the $i$ th particle are updated in each iteration according to

$$
\begin{aligned}
\mathbf{V}_{i}^{(l+1)}= & w * \mathbf{V}_{i}^{(l)}+\operatorname{rand}() * c_{1} *\left(\mathbf{P} \mathbf{b}_{i}^{(l)}-\check{\mathbf{P}}_{i}^{(l)}\right) \\
& +\operatorname{rand}() * c_{2} *\left(\mathbf{G} \mathbf{b}^{(l)}-\check{\mathbf{P}}_{i}^{(l)}\right) \\
\check{\mathbf{P}}_{i}^{(l+1)}= & \check{\mathbf{P}}_{i}^{(l)}+\mathbf{V}_{i}^{(l+1)}
\end{aligned}
$$

where $w$ is the inertia weight, $\operatorname{rand}()$ denotes the uniform random number between 0 and 1 , and $c_{1}$ and $c_{2}$ are the two acceleration coefficients. In order to avoid excessive roaming of particles beyond the search space [14], a velocity range

$$
\mathrm{V}=\left[-V_{\max }, V_{\max }\right]+j\left[-V_{\max }, V_{\max }\right]
$$

is imposed on each element of $\mathbf{V}_{i}^{(l+1)}$ so that

$$
\begin{aligned}
& \text { If }\left(\Re\left[\left.\mathbf{V}_{i}^{(l+1)}\right|_{p, q}\right]>V_{\text {max }}\right) \quad \Re\left[\left.\mathbf{V}_{i}^{(l+1)}\right|_{p, q}\right]=V_{\text {max }} ; \\
& \text { If }\left(\Re\left[\left.\mathbf{V}_{i}^{(l+1)}\right|_{p, q}\right]<-V_{\text {max }}\right) \quad \Re\left[\left.\mathbf{V}_{i}^{(l+1)}\right|_{p, q}\right]=-V_{\text {max }} ; \\
& \text { If }\left(\Im\left[\left.\mathbf{V}_{i}^{(l+1)}\right|_{p, q}\right]>V_{\max }\right) \quad \Im\left[\left.\mathbf{V}_{i}^{(l+1)}\right|_{p, q}\right]=V_{\text {max }} ; \\
& \text { If }\left(\Im\left[\left.\mathbf{V}_{i}^{(l+1)}\right|_{p, q}\right]<-V_{\max }\right) \quad \Im\left[\left.\mathbf{V}_{i}^{(l+1)}\right|_{p, q}\right]=-V_{\text {max }} ;
\end{aligned}
$$

where $\left.\mathbf{V}\right|_{p, q}$ denotes the $(p, q)$ th element of $\mathbf{V}$. Moreover, if the velocity (13) approaches zero, it is reinitialised to proportional to $V_{\max }$ with a small factor $\gamma$

$$
\left.\mathbf{V}_{i}^{(l+1)}\right|_{p, q}= \pm \operatorname{rand}() * \gamma *\left(V_{\max }+j V_{\max }\right) .
$$

Similarly, each element of $\check{\mathbf{P}}_{i}^{(l+1)}$ is checked to ensure that it stays inside the search space $\mathbf{S}$ :

$$
\begin{aligned}
& \text { If }\left(\Re\left[\left.\check{\mathbf{P}}_{i}^{(l+1)}\right|_{p, q}\right]>P_{\text {max }}\right) \\
& \quad \Re\left[\left.\check{\mathbf{P}}_{i}^{(l+1)}\right|_{p, q}\right]=\operatorname{rand}() * P_{\text {max }} ; \\
& \text { If }\left(\Re\left[\left.\check{\mathbf{P}}_{i}^{(l+1)}\right|_{p, q}\right]<-P_{\max }\right) \\
& \quad \Re\left[\left.\check{\mathbf{P}}_{i}^{(l+1)}\right|_{p, q}\right]=-\operatorname{rand}() * P_{\max } ; \\
& \text { If }\left(\Im\left[\left.\check{\mathbf{P}}_{i}^{(l+1)}\right|_{p, q}\right]>P_{\max }\right) \\
& \quad \Im\left[\left.\check{\mathbf{P}}_{i}^{(l+1)}\right|_{p, q}\right]=\operatorname{rand}() * P_{\max } ; \\
& \text { If }\left(\Im\left[\left.\check{\mathbf{P}}_{i}^{(l+1)}\right|_{p, q}\right]<-P_{\max }\right) \\
& \quad \Im\left[\left.\check{\mathbf{P}}_{i}^{(l+1)}\right|_{p, q}\right]=-\operatorname{rand}() * P_{\max } ;
\end{aligned}
$$

That is, if a particle is outside the search space, it is moved back inside the search space randomly, rather than forcing it to stay at the border [14]. 
d) Termination condition check. If the maximum number of iterations, $I_{\max }$, is reached, terminate the algorithm with the solution $\mathbf{G b}^{\left(I_{\max }\right)}$; otherwise, set $l=l+1$ and go to Step $b$ ).

It was reported in [13] that using a time varying acceleration coefficient (TVAC) enhances the performance of PSO. We adopt this mechanism, in which $c_{1}$ is reduced from 2.5 to 0.5 and $c_{2}$ varies from 0.5 to 2.5 during the iterative procedure according to

$$
\begin{aligned}
& c_{1}=(0.5-2.5) * l / I_{\max }+2.5, \\
& c_{2}=(2.5-0.5) * l / I_{\max }+0.5 .
\end{aligned}
$$

This TVAC mechanism works well in our application. We also remove the influence of the previous velocity by setting $w=0$, as suggested in [13]. Our empirical results suggest that the search limit can be set to $P_{\max }=1$ for our application. With this choice of position bound, the velocity limit can be set to $V_{\max }=1$. An appropriate value of the small factor $\gamma$ in (16) for avoiding zero velocity is found to be $\gamma=0.1$ by experiments. The computational complexity per iteration of this PSO based MBER MUT scheme is also listed in Table I.

\section{Simulation Results}

The BS employed $N=4$ transmit antennas to communicate over the $4 \times 4$ flat Rayleigh fading channels to $K=4$ single-receive-antenna QPSK mobile users. The size of the swarm was chosen to be $S=20$, which is a standard choice recommended in the literature for small to medium size optimisation problems. The maximum number of iterations for the PSO algorithm was set to $I_{\max }=20$, which was adequate as the PSO-based MBER-MUT algorithm with $I_{\max }=20$ and $S=20$ was seen to arrive at the same MBER performance also achieved by the SQP-based MBER-MUT design. All the simulation results were obtained by averaging over 100 channel realisations. First, perfect CSI knowledge was assumed at the BS, and Fig. 3 compares the BER performance of the MMSE-MUT scheme with that of the PSO-based MBERMUT scheme. It can be seen from Fig. 3 that the MBER-MUT

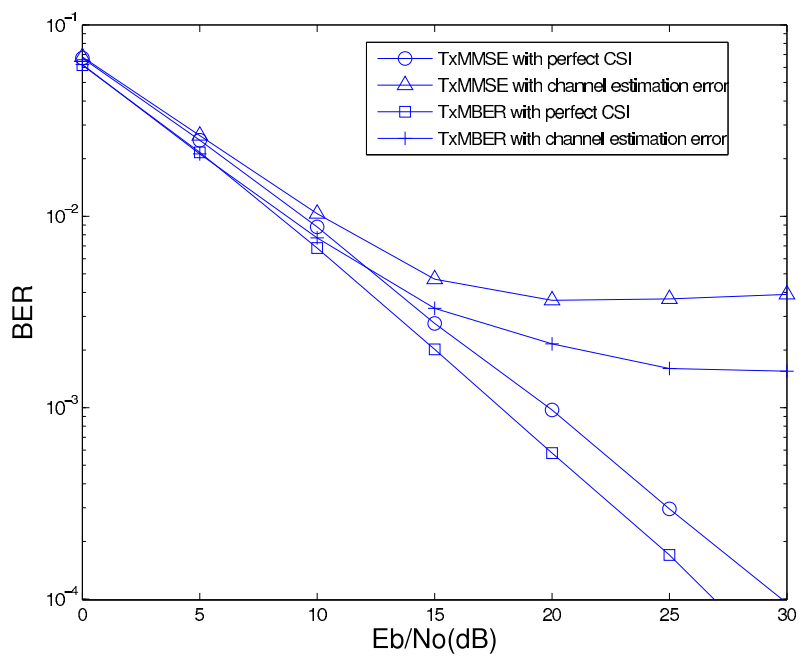

Fig. 3. BER versus SNR performance of the transmit MMSE and PSOaided MBER schemes, communicating over flat Rayleigh fading channels using $N=4$ transmit antennas to support $K=4$ QPSK users.

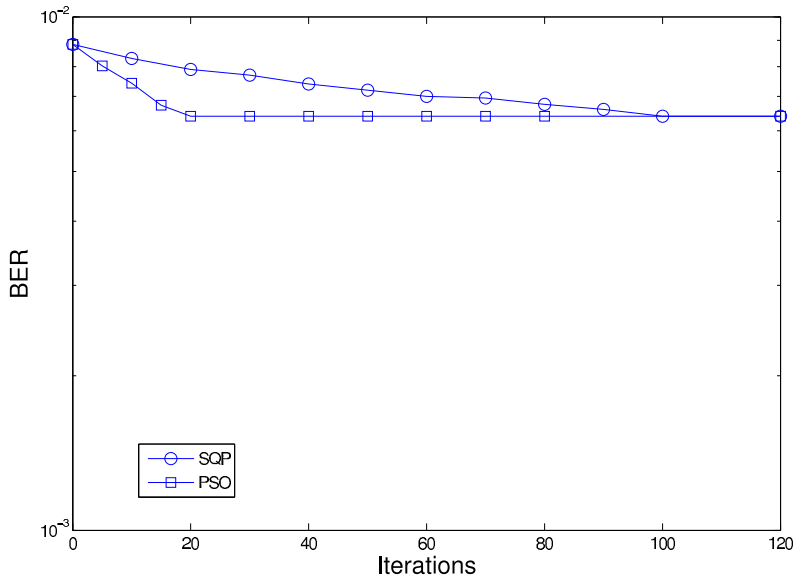

Fig. 4. Convergence performance of the SQP and PSO aided MBER-MUT schemes for the system employing $N=4$ transmit antennas to support $K=4$ QPSK mobile users over flat Rayleigh fading channels at $\mathrm{Eb} / \mathrm{No}=10 \mathrm{~dB}$.

achieved an SNR gain of $3 \mathrm{~dB}$ over the MMSE-MUT at the target BER of $10^{-4}$. The robustness of the two MUT schemes to channel estimation error was then investigated by adding a Gaussian white noise with a standard deviation of 0.05 to each channel tap $h_{i, k}$ to represent channel estimation error at the BS. The BERs of the MMSE-MUT and PSO-based MBERMUT schemes under this channel estimation error are also plotted in Fig. 3, where it can be seen that the MBER-MUT design was no more sensitive to channel estimation error than the MMSE-MUT design.

Complexity and convergence speed of the PSO-aided MBER-MUT were investigated, using the SQP-based MBERMUT as the benchmark. Given $\mathrm{SNR}=\mathrm{Eb} / \mathrm{No}=10 \mathrm{~dB}$, Fig. 4 compares the convergence performance of the SQP and PSO based MBER MUT schemes. It is clear from Fig. 4 that the SQP algorithm converged to the MBER-MUT solution after 100 iterations, while the PSO algorithm arrived at the same MBER-MUT solution with 20 iterations. Figs. 5 and 6

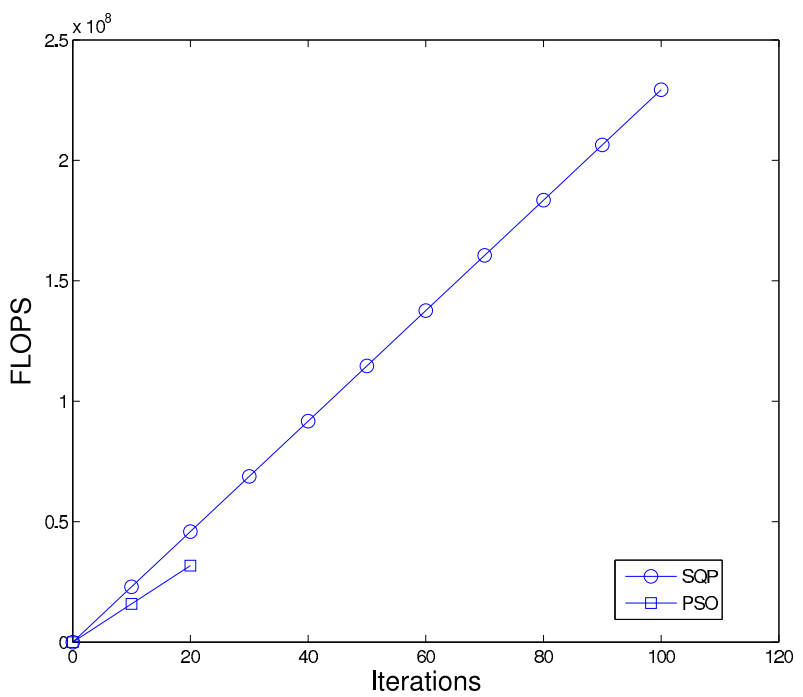

Fig. 5. Complexity of the SQP and PSO aided MBER-MUT schemes for the system employing $N=4$ transmit antennas to support $K=4$ QPSK mobile users over flat Rayleigh fading channels at $\mathrm{Eb} / \mathrm{No}=10 \mathrm{~dB}$. 


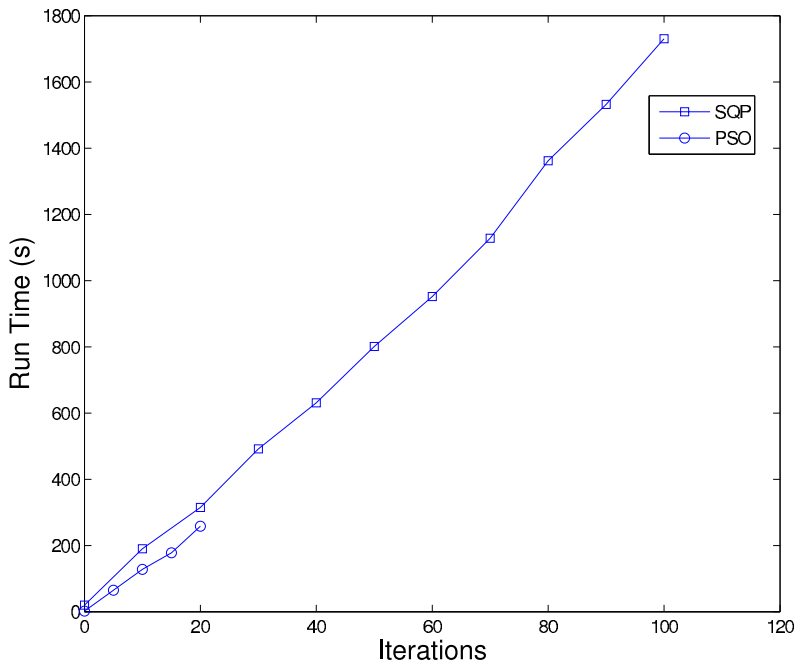

Fig. 6. Run times of the SQP and PSO aided MBER-MUT schemes for the system employing $N=4$ transmit antennas to support $K=4$ QPSK mobile users over flat Rayleigh fading channels at $\mathrm{Eb} / \mathrm{No}=10 \mathrm{~dB}$.

compare the computational requirements and run times of the SQP and PSO based MBER-MUT schemes over 100 channel realisations. It can be seen that the SQP algorithm needed $229,351,100$ Flops and costed $1730.6 \mathrm{~s}$ to converge to the MBER-MUT solution, while the PSO algorithm converged to the same MBER-MUT solution at the cost of 34,561,760 Flops and consumed $257.3 \mathrm{~s}$. Both the complexity and run time results agree that the PSO-aided MBER-MUT design imposed an approximately seven times lower complexity than the SQP counterpart for this system.

\section{CONCLUSIONS}

We have proposed a PSO assisted MBER-MUT algorithm, which offers a much lower computational complexity than the existing SQP based MBER-MUT algorithm. Specifically, our simulation results involving the system of four transmit antennas and four QPSK mobile users over flat Rayleigh fading channels have confirmed that the PSO-based MBERMUT imposes approximately seven times lower complexity than the SQP-based MBER-MUT.

\section{REFERENCES}

[1] T. M. Cover and J. A. Thomas, Elements of Information Theory. New York: John Wiley \& Sons, 1991.

[2] D. Yang, L.-L. Yang and L. Hanzo, "Performance of SDMA systems using transmitter preprocessing based on Noisy feedback of vector-quantized channel impulse responses," in Proc. VTC2007-Spring, (Dublin, Ireland), pp. 2119-2123, April 22-25, 2007.

[3] B. R. Vojčić and W. M. Jang, "Transmitter precoding in synchronous multiuser communications," IEEE Trans. Communications, vol. 46, pp. 1346-1355, October 1998 .

[4] R. Irmer, W. Rave and G. Fettweis, "Minimum BER transmission for TDD-CDMA in frequency-selective channels," in Proc. Int. Symp. Personal, Indoor And Mobile Radio Communications, vol. 2, (Beijing, China), pp. 1260-1264, Sept. 7-10, 2003.

[5] R. Irmer, R. Habendorf, W. Rave and G. Fettweis, "Nonlinear multiuser transmission using multiple antennas for TDD-CDMA," in Proc. 6th Int. Symp. Wireless Personal Multimedia Communications, (Yokosuka, Japan), pp. 251-255, Oct. 19-22, 2003.
[6] R. Irmer, R. Habendorf, W. Rave and G. Fettweis, "Nonlinear chip-level multiuser transmission for TDD-CDMA with frequency-selective MIMO channels," in Proc. 5th Int. ITG Conf. Source and Channel Coding, (Erlangen, Germany), pp. 363-370, Jan. 14-16, 2004.

[7] R. Irmer, Multiuser Transmission in Code Division Multiple Access Mobile Communication Systems. PhD thesis, Technique University of Dresden, Dresden, Germany, April 2005.

[8] A. Hjørungnes and P. S. R. Diniz, "Minimum BER prefilter transform for communications systems with binary signaling and known FIR MIMO channel," IEEE Signal Processing Letters, vol. 12, pp. 234-237, March 2005.

[9] S. Tan, Minimum Error Rate Beamforming Transceivers. PhD thesis, School of Electronics and Computer Science, University of Southampton, Southampton, UK, April 2008.

[10] J. Nocedal and S.J. Wright, Numerical Optimization. New York: Springer, 1999.

[11] J. Kennedy and R. Eberhart, "Particle swarm optimization," in Proc. of 1995 IEEE Int. Conf. Neural Networks, vol. 4, (Perth, Australia), pp. 1942-1948, Nov. 27 - Dec. 1, 1995.

[12] J. Kennedy and R. C. Eberhart, Swarm Intelligence. Morgan Kaufmann, 2001

[13] A. Ratnaweera, S. K. Halgamuge and H. C. Watson, "Self-organizing hierarchical particle swarm optimizer with time-varying acceleration coefficients," IEEE Trans. Evolutionary Computation, vol. 8, pp. 240255, June 2004.

[14] S. M. Guru, S. K. Halgamuge and S. Fernando, "Particle swarm optimisers for cluster formation in wireless sensor networks," in Proc. 2005 Int. Conf. Intelligent Sensors, Sensor Networks and Information Processing, (Melbourne, Australia), pp. 319-324, Dec. 5-8, 2005.

[15] H.-M. Feng, "Self-generation RBFNs using evolutional PSO learning," Neurocomputing, vol. 70, pp. 241-251, December 2006.

[16] F. A. Guerra and L. S. Coelho, "Multi-step ahead nonlinear identification of Lorenz's chaotic system using radial basis function neural network with learning by clustering and particle swarm optimiszation," Chaos, Solitons and Fractals, vol. 35, pp. 967-979, March 2008.

[17] W.-F. Leong and G. G. Yen, "PSO-based multiobjective optimization with dynamic population size and adaptive local archives," IEEE Trans. Systems, Man and Cybernetics, Part B, vol. 38, pp. 1270-1293, October 2008.

[18] Z. Lu and S. Yan, "Multiuser detector based on particle swarm algorithm," in Proc. 6th IEEE CAS Symp. Emerging Technologies: Frontiers of Mobile and Wireless Communication, vol. 2, (Shanghai, China), pp. 783-786, May 31 - June 2, 2004.

[19] Y. Zhao and J. Zheng, "Multiuser detection employing particle swarm optimization in space-time CDMA systems," in Proc. 2005 Int. Symp. Communications and Information Technology, vol. 2, pp. 940-942, Oct. 12-14, 2005.

[20] H. H. El-Mora, A. U. Sheikh and A. Zerguine, "Application of particle swarm optimization algorithm to multiuser detection in CDMA," in Proc. 16th IEEE Int. Symp. Personal, Indoor and Mobile Radio Communications, vol. 4, (Berlin, Germany), pp. 2522-2526, Sept. 11-14, 2005.

[21] Z. Guo, Y. Xiao and M. H. Lee, "Multiuser detection based on particle swarm optimization algorithm over multipath fading channels," IEICE Trans. Communications, vol. E90-B, pp. 421-424, 2007.

[22] K. K. Soo, Y. M. Siu, W. S. Chan, L. Yang and R. S. Chen, "Particleswarm-optimization-based multiuser detector for CDMA communications," IEEE Trans. Vehicular Technology, vol. 56, pp. 3006-3013, September 2007.

[23] H. Liu and J. Li, "A particle swarm optimization-based multiuser detection for receive-diversity-aided STBC systems," IEEE Signal Processing Letters, vol. 15, pp. 29-32, 2008.

[24] L. Hanzo, M. Münster, B. J. Choi and T. Keller, OFDM and MC-CDMA for Broadband Multi-User Communications, WLANs and Broadcasting. Chichester, UK: John Wiley, 2003.

[25] S. Chen, A. Livingstone, H.-Q. Du and L. Hanzo, "Adaptive minimum symbol error rate beamforming assisted detection for quadrature amplitude modulation," IEEE Trans. Wireless Communications, vol. 7, pp. 1140-1145, April 2008.

[26] S. Chen, A. Livingstone and L. Hanzo, "Minimum bit-error rate design for space-time equalization-based multiuser detection," IEEE Trans. Communications, vol. 54, pp. 824-832, May 2006. 